\title{
Si Nanopowder Based Anode Material for the Lithium Ion Battery Cell
}

\section{Aleksandr Morozov ${ }^{1,3, a}{ }^{*}$, Alexander Semencha ${ }^{2, b}$, Alexander Freidinn ${ }^{2,3, c}$, Wolfgang Müller ${ }^{3, d}$, Margarita Dronova ${ }^{2, e}$}

\author{
${ }^{1}$ Institute of Mechanics, Faculty of Mechanical Engineering, Berlin Institute of Technology, Berlin, \\ Germany \\ ${ }^{2}$ Peter the Great St. Petersburg Polytechnic University, St. Petersburg, Russia \\ ${ }^{3}$ Institute for Problems in Mechanical Engineering of Russian Academy of Sciences, St. \\ Petersburg, Russia
}

amorozov@tu-berlin.de, bvil-I@yandex.ru, calexander.freidin@gmail.com, dwolfgang.h.mueller@tu-berlin.de, emargarita-dronou@yandex.ru

Keywords: lithium ion battery, silicon based anode, chemical reaction front, chemical affinity tensor, linear viscoelasticity.

\begin{abstract}
We consider a silicon nanopowder based anode for a lithium ion battery cell. We present the design of the battery cell ready for in situ Raman and X-ray experiments and a technical procedure for the cell manufacturing. From the continuum mechanics point of view, this type of anode can be represented by a spherical nanoparticle surrounded by viscoelastic matrix. During the charging process this nanoparticle undergoes a chemical reaction. Based on the chemical affinity concept we describe how the mechanical properties of the matrix material influence the kinetics of the charging process. We study spherically symmetry problems numerically for different sets of matrix material parameters and show their influence on the reaction front kinetics.
\end{abstract}

\section{Introduction}

Lithium-Ion Batteries (LIBs) are important energy storage devices. They are required in various technological applications, such as portable electronics or for the powering of vehicles [1]. They show higher energy density, higher operating voltages, lower self-discharge, and lower maintenance requirements than any other types of rechargeable cells [2]. However, due to further miniaturization and other advances presently occurring in the portable device industry, and in order to use them advantageously for aerospace, military, and automotive applications, their mass capacities and their energy densities require a further increase. There is a considerable interest in developing next generation LIBs with high specific capacity. This goal can be reached by replacing the already widelyused lithium cobalt oxide cathodes and carbonaceous anodes with other higher performance electrode materials.

Among all candidates for anode materials silicon ( $\mathrm{Si}$ ) has currently the highest capacity for reversible Li intercalation. Also, $\mathrm{Si}$ is the second most abundant element on Earth and there is already a mature industrial infrastructure in existence, which also adds to its attractiveness when considering commercial applications [3]. For the particular case of silicon, the (theoretical) compound formed after maximum charging is $\mathrm{Li} 4.4 \mathrm{Si}$ (Li22 $\mathrm{Si}$ ). It corresponds to the highest known theoretical charge capacity $(>3500 \mathrm{mAh} / \mathrm{g})$, which is about 10 times higher than the charge capacity of graphite anodes, which are mostly used (about $372 \mathrm{mAh} / \mathrm{g}$ ). Silicon can host up to $4.4 \mathrm{Li}$ atoms per $\mathrm{Si}$ atom reversibly compared to one Li atom hosted by only by 6 carbon atoms. However, the alloying and de-alloying with large amounts of $\mathrm{Li}$ results in dramatic volumetric $300 \%$ changes in $\mathrm{Si}$ anodes. In order to cope with the large volume change and, therefore, in order to obtain better capacity retention and cycle life for Si anodes, various designs of different anode structures have been proposed, such as thin films [4], nanoparticles [5], nanowires and hollow nanowires [6,7], and even remarkable morphologies, such as honeycomb structures [8]. Although large improvements have been made experimentally by nano-structuring, the material integrity, and hence the lifetime, remain a critical issue [9]. In addition, 
cycle stability of silicon anodes is strongly dependent on geometrical design. Also, a quantitative study of the effect of lithiation-associated stress on the driving force of lithiation is still pending [6].

One of the alternative host materials to $\mathrm{Si}$ are synthesized WSe2 nanoparticles which were used as anodes to assemble lithium-ion batteries in [10]. A high reversible capacity and very stable cycle performance can be obtained, implying that these nanostructures could provide the new types of electrode materials for rechargeable lithium-ion batteries. Nevertheless, volume expansion during lithiation is also immanent in this material. However, the details of the compound formation are still under discussion and subject to ongoing research [11].

In the next section the design of the battery cell ready for in situ Raman and X-ray experiments is described, the technical procedure for the manufacturing is discussed, and a cycling test of the produced specimen is shown. Afterwards, a mechanical model for the charging process of the $\mathrm{Si}$ particle in matrix media is presented. The problem of chemical reaction front propagation in a spherical particle surrounded by a linear viscoelastic material is solved numerically. The influence of the matrix mechanical properties on the kinetics of the chemical reaction front is highlighted. At the end, conclusions and an outlook are formulated.

\section{Battery Cell Preparation}

The cells were assembled using an experimental anode, Li foil as the cathode, a separator soaked in electrolyte and $\mathrm{Ni}$ current collectors all sealed in a transparent polyester bag. It is a common procedure to use metallic $\mathrm{Li}$ in electrochemical studies of the anode materials $[12,13,14]$. This allows to avoid any limitations of Li-ion concentration in the system [15]. The schematic electrochemical cell is shown in Fig. 1.

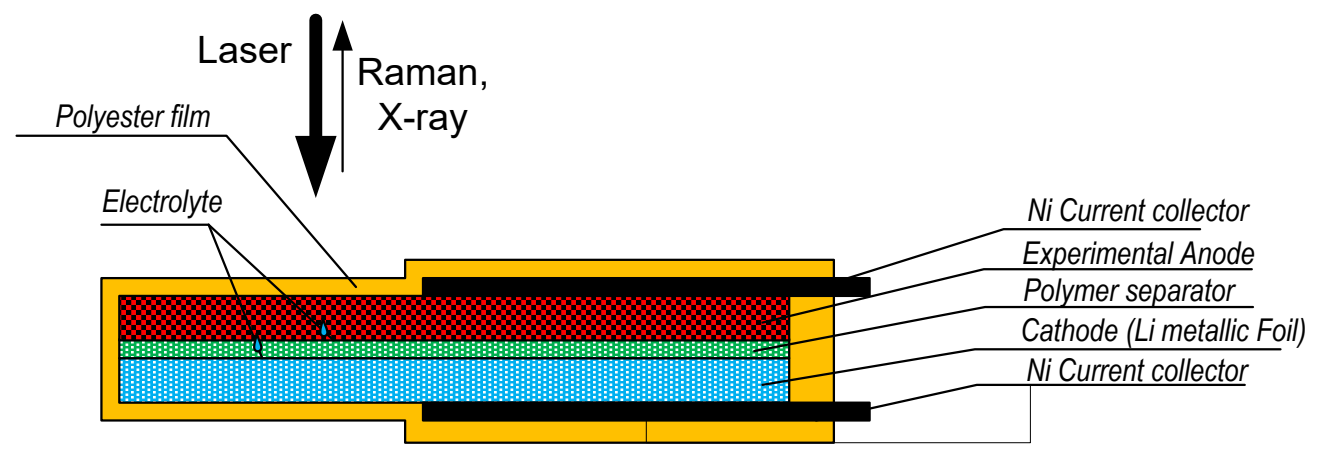

Figure 1. Schematic representation of the anode battery cell.

Two anode structures are considered in the battery cell. The first one (Fig. 2, left) is designed for performing Raman microscopy measurements. In this design the experimental anode material covers the stainless-steel mesh foil, which is used to homogenize the electron distribution and therefore to homogenize the charging process along the anode. The anode material covers the mesh from both sides, so that there is an access for the Raman beam to evaluate the mechanical strains in the anode in-situ by measuring the peak shift [16]. The second type of anode structure (Fig. 2, right) is designed for the X-ray diffraction experiment [17]. It consists of a thin layer of anode material put on the Be foil, which is transparent for X-rays and widely used, i.e., in X-ray detectors in various scanners $[18,19]$.

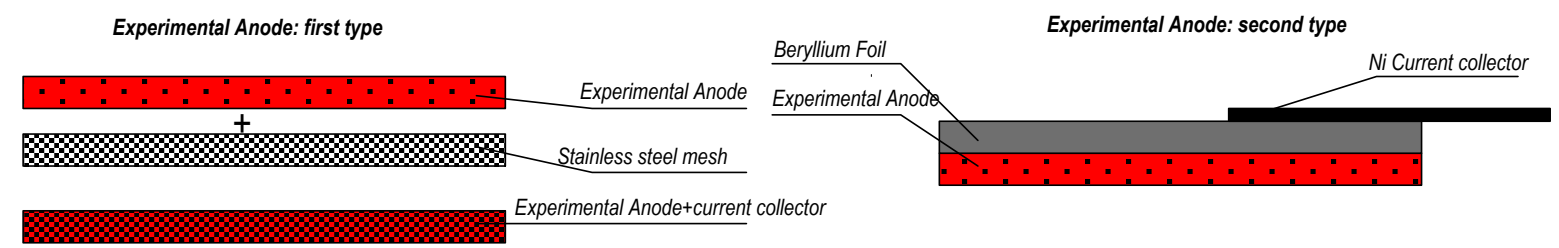

Figure 2. Schematic representation of different anode designs. 
For the first test a set of battery cells with the design shown in Fig. 2, right, were assembled. However, not by using $\mathrm{Be}$, but $\mathrm{Cu}$ foil. The active mass of the experimental anode consists of $\mathrm{Si}$ nanoparticles (mean diameter $100 \mathrm{~nm}$ ), which are the main carrier of the cell capacity, conductive carbon, which is used to gain the conductivity of the anode, and carboxymethyl cellulose as a binder. Materials were mixed with a mass ratio 50:40:10, respectively, and stirred in deionized water. The amount of water was adjusted to reach a viscosity $\sim 5000 \mathrm{cP}$. The slurry was put on the thin $\mathrm{Cu}$ film and dried in a vacuum oven for $3 \mathrm{~h}$ at $90^{\circ} \mathrm{C}$. The cells were assembled in an Ar-filled glovebox. The level of oxygen during the assembly was less than $1 \%$ and controlled by an SST oxygen sensor.

A photo of the assembled battery cell is shown in Fig. 3, left. A battery cycling test was performed to determine the quality of the specimen. In Fig. 3, right, the voltage (red curve) and current (blue curve) are shown as a function of time during the cycling. The battery cell provides the estimated voltage level.
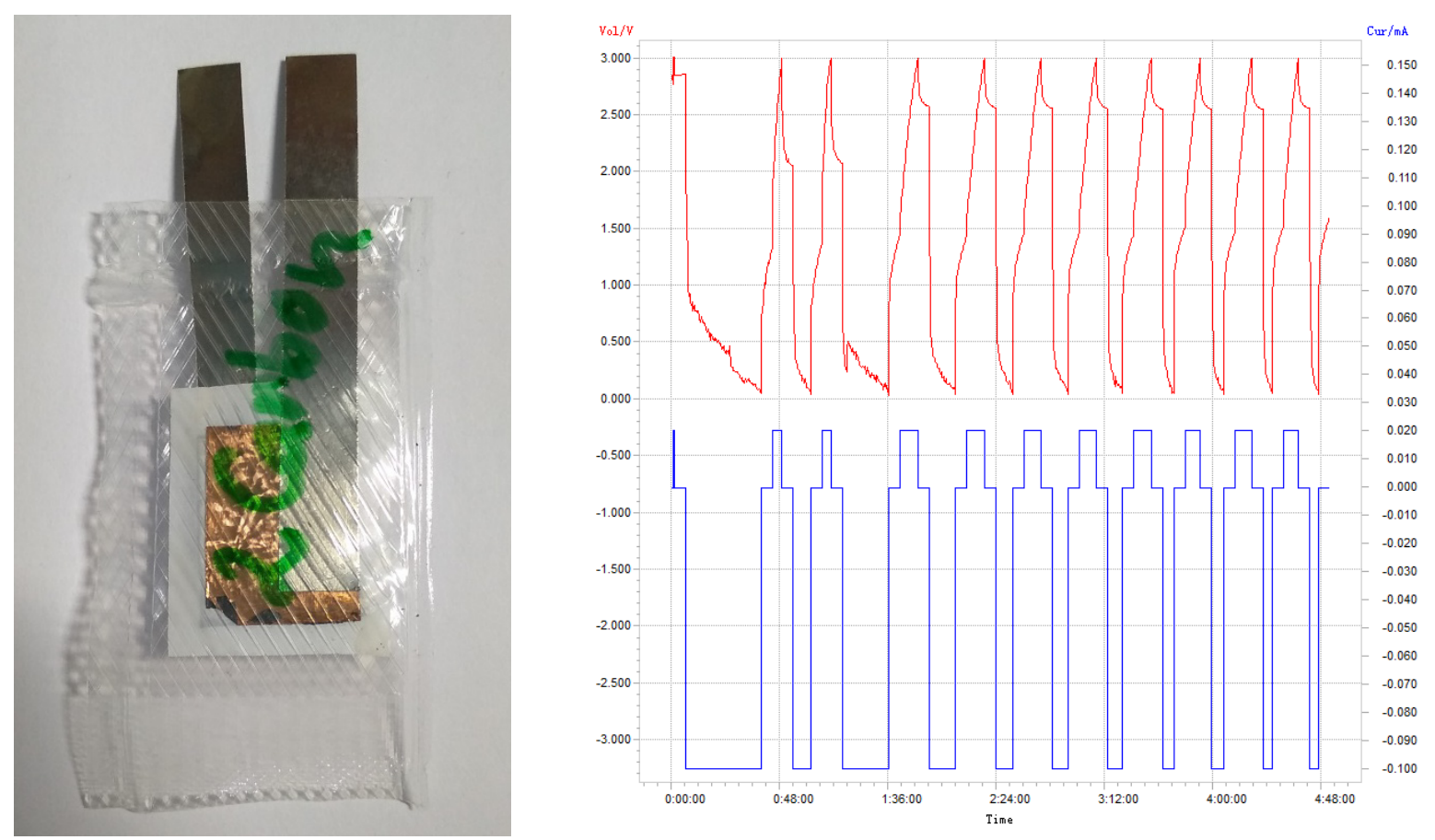

Figure 3. Battery cell (left) and its cycling test results (right).

\section{Reaction Front Kinetics in Deformable Solids}

In the case of the charging process in LIBs we consider a reaction between Li-ions and Si localized at the sharp interface and sustained by $\mathrm{Li}$-ions diffusion through $\mathrm{Li}_{\mathrm{x}} \mathrm{Si}$. In general, this reaction can be written as follows,

$$
n_{-} B_{-}+n_{*} B_{*} \rightarrow n_{+} B_{+}
$$

where the index "-" denotes the initial material $(\mathrm{Si})$, " $*$ " is for diffusive constituent $\left(\mathrm{Li}^{+}\right)$supplied from the external environment, and "+" stands for the product of the chemical reaction $\left(\mathrm{Li}_{\mathrm{x}} \mathrm{Si}\right), n_{k}$ are the stoichiometric coefficients and $B_{k}$ refer to the chemical symbols of the reactants.

From the mass balance on the interface one can express the chemical reaction front velocity through the reaction rate, $\omega$,

$$
W=\frac{n_{-} M_{-} \omega}{\rho_{-}}
$$

where the normal, $\mathbf{N}$, is pointing outwards of the domain occupied by the material "+".

As mentioned earlier, various experimental observations show that mechanical stresses can affect the chemical reaction rate. In this paper, a chemical affinity tensor concept [20] is used in order to couple the chemical reaction rate, $\omega$, and the mechanical stresses raised due to the transformation strains 


$$
\omega=k_{*} c\left[1-\exp \left(-\frac{A_{N N}}{R T}\right)\right],
$$

where $k_{*}$ is a reaction constant, $c$ is the concentration of the diffusive constituent at the reaction front, $R$ is the ideal gas constant, $T$ is absolute temperature, and $A_{N N}=\mathbf{N} \cdot \mathbf{A} \cdot \mathbf{N}$ is the normal component of the chemical affinity tensor. According to this concept, the chemical affinity of solid bodies is considered as a tensorial quantity, meaning that the chemical reaction occurs on the oriented surface of the material.

In the quasistatic case the chemical affinity tensor can be defined as a linear combination of chemical potentials, which are tensorial quantities (see the discussions in [21] and the references therein), weighted by the stoichiometric coefficients, $n_{k}$, and the molar masses, $M_{k}$. The chemical affinity tensor takes the form $[20,22]$

$$
\mathbf{A}=n_{-} M_{-} \mathbf{M}_{-}+n_{*} M_{*} \mu_{*} \mathbf{I}-n_{+} M_{+} \mathbf{M}_{+}
$$

where $\mu_{*}$ is the chemical potential of the diffusive constituent and $\mathbf{M}_{ \pm}$are tensorial chemical potentials of solids, which can be represented by Eshelby energy momentum tensors weighted by the referential mass densities,

$$
\mathbf{M}_{ \pm}=f_{ \pm} \mathbf{I}-\frac{1}{f_{ \pm}} \mathbf{F}_{ \pm}^{T} \cdot \mathbf{S}_{ \pm}
$$

where $f_{ \pm}$are the specific Helmholtz free energy densities of the solids involved in the reaction, $\mathbf{F}$ is the deformation gradient, $\mathbf{I}$ is the identity tensor and $\mathbf{S}$ is the first Piola-Kirchhoff stress tensor. The chemical potential of the diffusive constituent can be taken in the following form,

$$
M_{*} \mu_{*}=\eta_{*}(T)+R T \ln \frac{c}{C_{*}},
$$

where $\eta_{*}(T)$ is a temperature dependent chemical energy of the diffusive constituent, $c$ is a concentration of the diffusive constituent at the reaction front and $c_{*}$ the concentration in a reference concentration.

\section{Spherical particle in linear viscoelastic media}

We consider a chemical reaction in a spherical particle placed in a finite isotropic viscoelastic medium fixed at the outer boundary, Fig. 4. Both initial and chemically transformed materials are assumed to be isotropic and linearly elastic. The chemical reaction starts from the sphere's boundary and is sustained by the diffusion of the gaseous constituents through the transformed material. It is assumed that the diffusive constituent freely moves through the matrix material so that a sufficient supply for the diffusion process in the particle is provided. In addition, a Fickean model of the diffusion is considered, assuming that the diffusion process is much faster than chemical reaction front propagation and strain relaxation.

In the particle the Cauchy stresses are related to the strains via Hooke's law,

$$
\sigma_{-}=\mathrm{C}_{-}: \varepsilon_{-}, \quad \sigma_{+}=\mathrm{C}_{+}:\left(\varepsilon_{+}-\varepsilon^{\mathrm{ch}}\right),
$$

where $\mathbf{C}_{+}, \mathbf{C}_{-}$are elasticity tensors for the solid constituents and $\varepsilon^{\text {ch }}=\theta_{\text {ch }} \mathbf{I}$ is a chemical transformation strain tensor. The case of large deformations and non-linear viscoelastic materials for the Si particle charging model was previously studied numerically in [23].

For the matrix media, a Kelvin-Voigt rheology model of viscoelasticity is assumed with the constitutive relation

$$
\boldsymbol{\sigma}_{-}=\mathbf{C}_{\mathrm{m}}: \boldsymbol{\varepsilon}_{-}+2 \eta \dot{\boldsymbol{e}}_{\mathrm{m}}
$$

where $\eta$ is a viscosity parameter, and $\boldsymbol{e}_{\mathrm{m}}$ is a deviatoric part of the strain tensor. A similar material model was studied analytically for the spherical symmetric problems in [24,25]. 


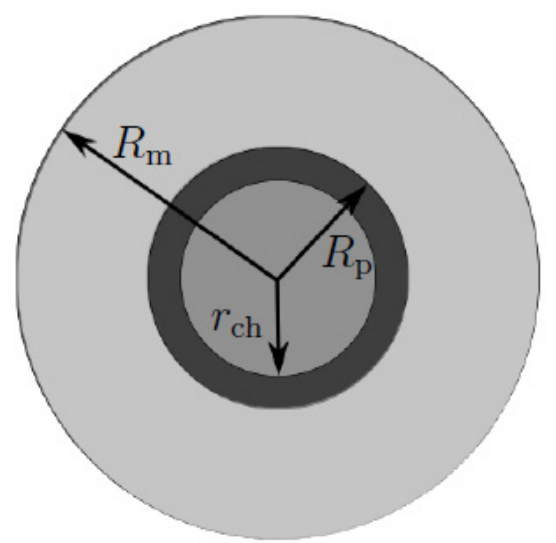

Figure 4. Spherical particle with radius $R_{\mathrm{p}}$ surrounded by a spherical medium $R_{\mathrm{m}}$. The reaction front assumed to be spherical and defined by $r_{\mathrm{ch}}$.

For a spherical particle such that the elastic materials undergo a chemical transformation the normal component of the affinity tensor is derived as a function of mean strains $\varepsilon_{-}$[26],

$$
A_{N N}=\frac{n_{-} M_{-}}{\rho_{-}} \chi\left(\varepsilon_{-}\right)+n_{*} R T \ln \frac{c}{c_{*}},
$$

where $K_{+}, K_{-}$are the bulk moduli, $\mu_{+}, \mu_{-}$are shear moduli, jump $\llbracket K \rrbracket=K_{+}-K_{-}$, and

$$
\chi\left(\varepsilon_{-}\right)=\gamma-\frac{2 K_{+} \mu_{+}}{3 K_{+}+4 \mu_{-}} \theta_{\mathrm{ch}}^{2}-\frac{3}{2} \cdot \frac{3 K_{-}+4 \mu_{+}}{3 K_{+}+4 \mu_{-}}\left(3 \llbracket K \rrbracket \varepsilon_{-}-2 K_{+} \theta_{\mathrm{ch}}\right) \varepsilon_{-} .
$$

In order to specify a kinetic equation for the chemical reaction front propagation, one has to find the strains and the concentration of the diffusive constituent at the reaction front. This problem involves two time dependent processes: the movement of the interface and a strain relaxation due to the viscosity of the matrix material. The corresponding solution cannot be found in a closed form, hence a time integration is performed numerically by using an explicit Euler scheme. In the case of $n_{*}=1$, the reaction front velocity takes the form

$$
W=-\dot{r}_{\mathrm{ch}}=\frac{n_{-} M_{-}}{\rho_{-}} k_{*}\left(c-c_{\mathrm{eq}}\right),
$$

where $\dot{r}_{\mathrm{ch}}$ is a current fixed position of the chemical interface radius, and the equilibrium concentration [27], at which thermodynamic force is equal to zero, is given by

$$
c_{\mathrm{eq}}=c_{*} \exp \left(-\frac{n_{-} M_{-}}{\rho_{-}} \cdot \frac{\chi\left(\varepsilon_{-}\right)}{R T}\right) .
$$

Radius of the interface at $i^{\text {th }}$ iteration

$$
r_{\mathrm{ch}}^{i+1}=r_{\mathrm{ch}}^{i}-\frac{n_{-} M_{-}}{\rho_{-}} k_{*}\left[c\left(r_{\mathrm{ch}}^{i}\right)-c_{\mathrm{eq}}\left(\varepsilon_{-}{ }^{i}, t^{i}\right)\right] \mathrm{d} t
$$

where $\mathrm{d} t=t^{i+1}-t^{i}$ is a constant time increment.

\section{Results}

Material and diffusion parameters used in the simulation are given in Tables 1 and 2, respectively.

Table 1. Material properties and parameters used in the analytical and numerical simulation. Material "+" refers to the outer material of the particle, material "-" to the inner, respectively.

\begin{tabular}{|c|c|c|c|}
\hline & Material “-” & Material “-” & Matrix material \\
\hline$E[\mathrm{~Pa}]$ & 80 & 60 & 40 \\
\hline$v$ & 0.22 & 0.22 & 0.38 \\
\hline$\eta[\mathrm{Pa} \cdot \mathrm{s}]$ & N/A & N/A & 2 \\
\hline
\end{tabular}


Table 2. Diffusion properties and material parameters used in analytical and numerical simulation.

\begin{tabular}{|c|c|c|c|c|}
\hline & $k_{*} c_{*}\left[\frac{\mathrm{mol}}{\mathrm{mm}^{2} \mathrm{~s}}\right]$ & $D\left[\mathrm{~mm}^{2} / \mathrm{s}\right]$ & $\alpha c_{*}\left[\frac{\mathrm{mol}}{\mathrm{mm}^{2} \mathrm{~s}}\right]$ & $\frac{n_{-} M_{-}}{\rho_{-}}\left[\frac{\mathrm{mm}^{3}}{\mathrm{~mol}}\right]$ \\
\hline Value & $10^{-3}$ & $10^{-1}$ & $10^{-2}$ & $6.3 \cdot 10^{-2}$ \\
\hline
\end{tabular}

The following results are presented in dimensionless form, which is for time $\tau=\frac{E_{\mathrm{m}} t}{\eta}$, for the interface radius $\rho=\frac{r_{\mathrm{ch}}}{R_{\mathrm{p}}}$, and for the interface velocity $v=W \frac{\eta}{E_{\mathrm{m}} R_{\mathrm{p}}}$.

To demonstrate the kinetics, the position of the reaction front as a function of time and the interface velocity as a function of front position are shown in Fig. 5 and 6 respectively.

Black dotted curves in these figures stands for the case of rigid matrix material. In this case the input of stresses becomes compatible with the input of chemical energies and diffusion for given set of parameters. As a result, the driving force becomes zero and the reaction is stopped at a certain radius. For this configuration the velocity of the reaction front decreases from initial to zero value. An opposite case, when the matrix is a purely elastic material, is shown by the blue dashed curves. The stresses have less influence to the driving force, and so the reaction front reaches the center of the particle with increasing velocity.

Results for the configuration with viscoelastic matrix material are shown in the red solid curves. At first, the velocity of the reaction front is higher than that of the stress relaxation, so velocity of the front decreases as in the case of rigid matrix. When the front slows down, the influence of relaxation becomes more important. As a result, velocity increases again, and the particle transforms fully.

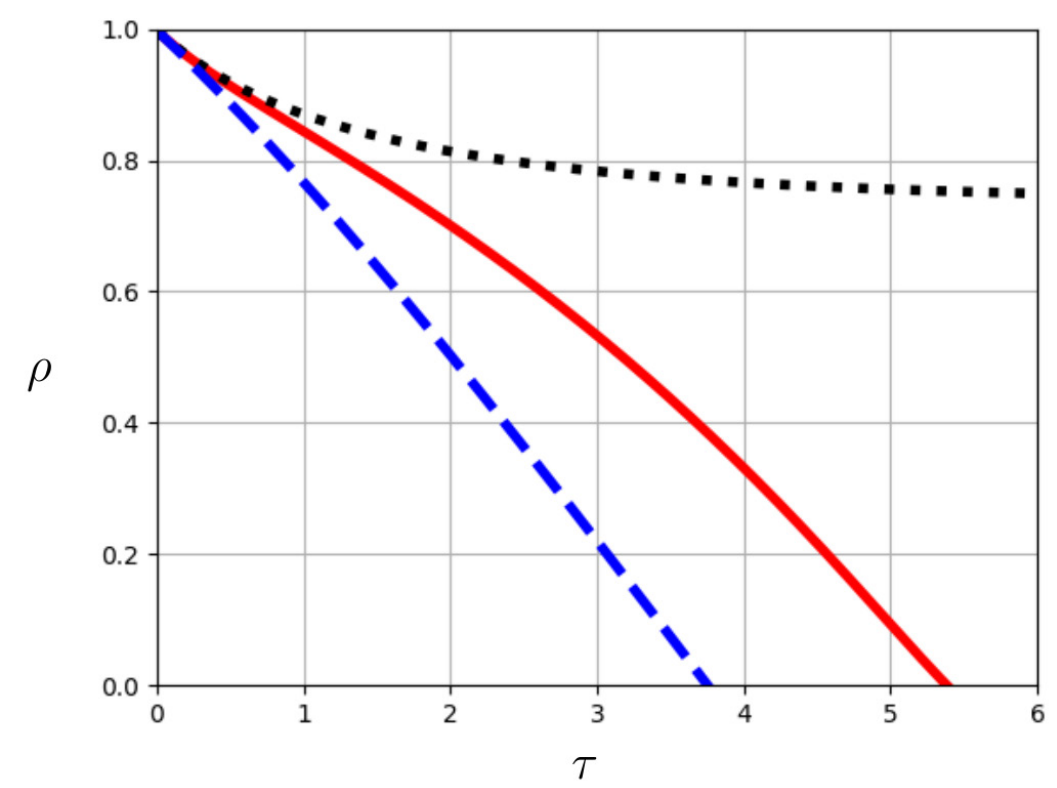

Figure 5. Dimensionless position of the reaction front as a function of normalized time for the case of rigid (black, dotted), elastic (blue, dashed) and linear viscoelastic (red, solid) matrix material. 


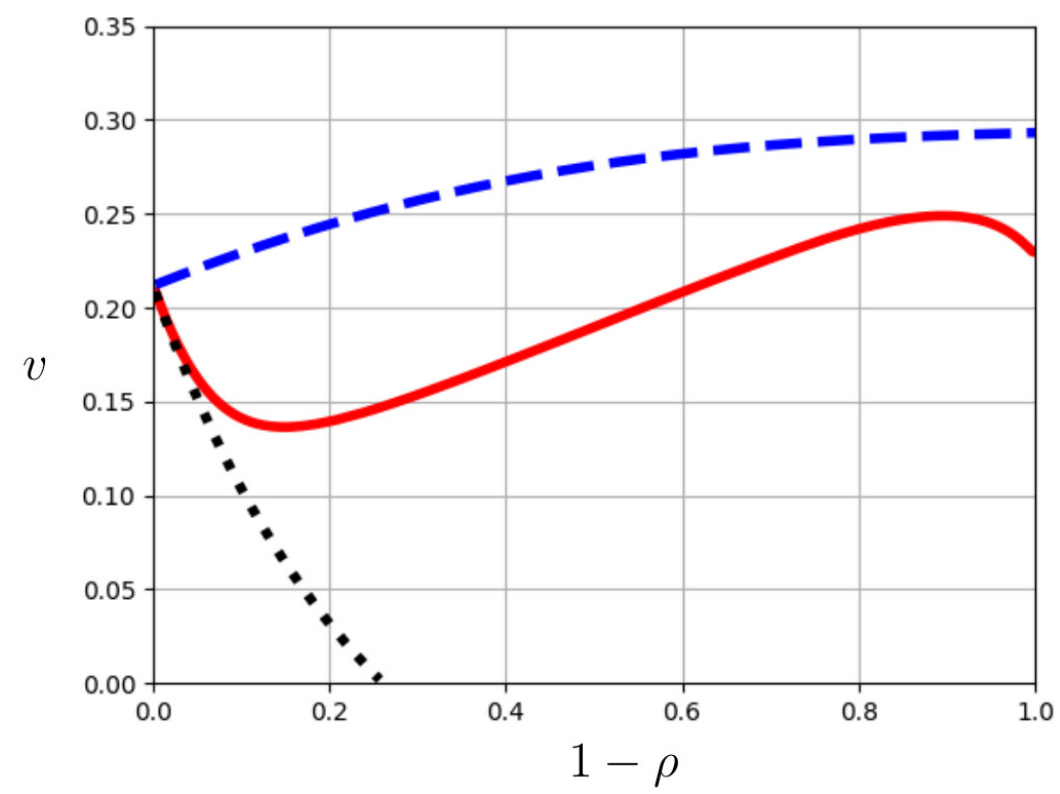

Figure 6. Normalized velocity of the reaction front as a function of its position for the case of rigid (black, dotted), elastic (blue, dashed) and linear viscoelastic (red, solid) matrix material.

\section{Conclusion}

We presented first results devoted to the design of the battery cell for Raman and X-ray experiments and the procedure of the cell manufacturing. For Raman we will use an anode design based on the stainless-steel mesh, and for the X-ray diffraction study Be film-based anodes.

In the active anode mass, the main capacitor are Si nanoparticles mixed and stirred in the viscous cellulose material. Therefore, we modelled a single particle charging process depending on the matrix material properties. We showed that reaction can accelerate, retard or have a more complex behavior depending on chosen parameters.

\section{Acknowledgments}

Authors appreciate the support of the joint DFG/RFBR grants (No. MU 1752/47-1 and 17-5112055).

\section{References}

[1]. M. Maximov, P. Novikov, A. Rumyantsev, Y. Koshtyal, D. Nazarov, A. Silin, A. Popovich, Application of atomic layer deposition in Li-ion batteries, NANOCON 2016 - Conference Proceedings, 8th International Conference on Nanomaterials - Research and Application 2016, 296302.

[2]. U. Kasavajjula, C. Wang, A.J. Appleby, Nano- and bulk-silicon-based insertion anodes for lithium-ion secondary cells, Journal of Power Sources 163 (2007) 1003-1039.

[3]. R. Ruffo, S.S. Hong, C.K. Chan, R.A. Huggins, Y. Cui, Impedance analysis of silicon nanowire lithium ion battery anodes, The Journal of Physical Chemistry C. 113 (2009) 11390-11398. [4]. S. Bourderau, T. Brousse, D. Schleich, Amorphous silicon as a possible anode material for Li-ion batteries, Journal of Power Sources 81-82 (1999) 233-236.

[5]. X.H. Liu, J.W. Wang, S. Huang, F. Fan, X. Huang, Y. Liu, S. Krylyuk, J. Yoo, S.A. Dayeh, A.V. Davydov, S.X. Mao, S.T. Picraux, S. Zhang, J. Li, T. Zhu, J.Y. Huang, In situ atomic-scale imaging of electrochemical lithiation in silicon, Nature Nanotechnology 7 (2012) 749-756.

[6]. Z. Jia, T. Li, Stress-modulated driving force for lithiation reaction in hollow nano-anodes, Journal of Power Sources 275 (2015) 866-876. 
[7]. K. Zhao, M. Pharr, L. Hartle, J.J. Vlassak, Z. Suo, Fracture and debonding in lithium-ion batteries with electrodes of hollow core shell nanostructures, Journal of Power Sources 218 (2012) 6-14.

[8]. L. Baggetto, D. Danilov, P.H.L. Notten, Honeycomb-structured silicon: remarkable morphological changes induced by electrochemical (de)lithiation, Advanced Materials 23 (2011) 1563-1566.

[9]. A.A. Popovich, M.Y. Maximov, P.A. Novikov, A.O. Silin, D.V. Nazarov, A.M. Rumyantsev, Cyclic stability of the anode material based on tin(IV) oxide for thin-film current sources, Russian Journal of Applied Chemistry 89 (2016) 679-681

[10]. E. Vasilyeva, A. Nasibulin, O. Tolochko, A. Rudskoy, A. Sachdev, X. Xiao, Application of $\mathrm{WSe}_{2}$ nanoparticles synthesized by chemical vapor condensation method for li-ion battery anodes, Zeitschrift für Physikalische Chemie 229 (2015) 1429-1437.

[11]. E. Vasilyeva, A. Nasibulin, O. Tolochko, A. Rudskoy, A. Sachdev, X. Xiao, Synthesis of tungsten diselenide nanoparticles by chemical vapor condensation method, Materials Science 21 (2015) 339-342.

[12]. H. Liu, D. Su, G. Wang, S.Z. Qiao, An ordered mesoporous $\mathrm{WS}_{2}$ anode material with superior electrochemical performance for lithium ion batteries, J. Mater. Chem. 22 (2012) 17437-17440.

[13]. D. Fenske, A. Eichhöfer, Nanostructured transition metal chalcogenides as materials in lithium ion batteries, Institute of Nanotechnology, KIT, p. 17.

[14]. P. Lavela, J. Morales, L. Sánchez, J.L. Tirado, Novel layered chalcogenides as electrode materials for lithium-ion batteries, Journal of Power Sources 68 (1997) 704-707.

[15]. T. Piao, S.-M. Park, C.-H. Doh, S.-I. Moon, Intercalation of lithium ions into graphite electrodes studied by AC impedance measurements, Journal of The Electrochemical Society 146 (1999) 2794-2798.

[16]. Z. Zeng, N. Liu, Q. Zeng, S. Woo Lee, W.L. Mao, C. Yi, In situ measurement of lithiationinduced stress in silicon nanoparticles using micro-Raman spectroscopy, Nano Energy 22 (2016) $105-110$.

[17]. S. Tardif, E. Pavlenko, L. Quazuguel, M. Boniface, M. Marechal, J.-S. Micha, L. Gonon, V. Mareau, G. Gebel, P. Bayle-Guillemaud, F. Rieutord, S. Lyonnard, Operando Raman spectroscopy and synchrotron X-ray diffraction of lithiation/delithiation in silicon nanoparticle anodes, ACS Nano 11 (2017) 11306-11316.

[18]. V.V. Mishin, I.A. Shishov, Investigation of deformation and fracture for thin beryllium foils under static loading by external pressure, Materials Physics and Mechanics 36 (2018) 100-113.

[19]. V.V. Mishin, I.A. Shishov, A. Minchena, Technique of durability estimation for thin beryllium foils during their work in X-ray detectors, Materials Physics and Mechanics 38 (2018) 4047.

[20]. A.B. Freidin, Chemical affinity tensor and stress-assist chemical reactions front propagation in solids, In: Proceedings of the ASME 2013 International Mechanical Engineering Congress and Exposition, vol. 9, p. V009T10A102, American Society of Mechanical Engineers (2013).

[21]. M. Grinfeld, Thermodynamic methods in the theory of heterogeneous systems. Longman Sc \& Tech, 1991.

[22]. A.B. Freidin, On chemical reaction fronts in nonlinear elastic solids. In: D. Indeitsev; A. M. Krivtsov, eds., Proceedings of XXXVII International Summer School-Conference Advanced Problems in Mechanics (2009) 231- 237.

[23]. M. Poluektov, A.B. Freidin, L. Figiel, Modelling stress-affected chemical reactions in nonlinear viscoelastic solids with application to lithiation reaction in spherical Si particles, International Journal of Engineering Science 128 (2018) 44-62.

[24]. K.P. Frolova, E.N. Vilchevskaya, W.H. Müller, W. Weiss, Comparison of numerical approaches for inverse Laplace transform by the example of intraocular pressure determination, In Proceedings of the International Summer School-Conference APM-2016, 2016, pp. 126-138. 
[25]. S.M. Bauer, K.P. Frolova, E.N. Vilchevskaya, Determination of the shear viscosity of sclera, In Advanced Problems in Mechanics, XLIV International Summer School-Conference Book of Abstracts, 2016, pp. 49.

[26]. A. Freidin, N. Morozov, S. Petrenko, E. Vilchevskaya, I. Korelev, Chemical reactions in spherically-symmetric problems of mechanochemistry, Acta Mechanica 227 (2015) 43-56.

[27]. A.B. Freidin, E.N. Vilchevskaya, I. Korolev, Stress-assist chemical reactions front propagation in deformable solids, International Journal of Engineering Science 83 (2014) 57-75. 